\title{
Robust Optimal Sliding-Mode Tracking Control for a Class of Uncertain Nonlinear MIMO Systems
}

\author{
Haiping Pang ${ }^{1}$ and Xiuqin Yang ${ }^{2}$ \\ ${ }^{1}$ College of Automation and Electronic Engineering, Qingdao University of Science and Technology, Qingdao 266042, China \\ ${ }^{2}$ Naval Aviation Engineering Institute of Qingdao Branch, Qingdao 266041, China \\ Correspondence should be addressed to Haiping Pang; panghp123@163.com
}

Received 27 April 2013; Revised 19 September 2013; Accepted 23 September 2013

Academic Editor: Weihai Zhang

Copyright (c) 2013 H. Pang and X. Yang. This is an open access article distributed under the Creative Commons Attribution License, which permits unrestricted use, distribution, and reproduction in any medium, provided the original work is properly cited.

\begin{abstract}
This paper addresses the problem of tracking a reference trajectory asymptotically given by a linear time-varying exosystem for a class of uncertain nonlinear MIMO systems based on the robust optimal sliding-mode control. The nonlinear MIMO system is transformed into a linear one by the input-output linearization technique, and at the same time the input-output decoupling is realized. Thus, the tracking error equation is established in a linear form, and the original nonlinear tracking problem is transformed into an optimal linear quadratic regulator (LQR) tracking problem. A LQR tracking controller (LQRTC) is designed for the corresponding nominal system, and the integral sliding-mode strategy is used to robustify the LQRTC. As a result, the original system exhibits global robustness to the uncertainties, and the tracking dynamics is the same as that of LQRTC for the nominal system. So a robust optimal sliding-mode tracking controller (ROSMTC) is realized. The proposed controller is applied to a two-link robot system, and simulation results show its effectiveness and superiority.
\end{abstract}

\section{Introduction}

Trajectory tracking control for multiple-input multipleoutput (MIMO) nonlinear systems has attached much attention during the past decades $[1,2]$. Compared with singleinput single-output (SISO) systems, the optimal tracking control for MIMO systems is much more difficult and complex because the output variables are more than one and usually coupled. Many real nonlinear plants have MIMO structures, such as robots, electric motors, and aerocrafts. The key to solve MIMO problem is to introduce the decoupling technology, and several control schemes for decoupling have been quite mature, such as the cascade decoupling based on classical control theory [3], the linear state feedback decoupling based on modern control theory [4], the linear output feedback decoupling [5], the stable-state feedback decoupling, and the dynamic precompensate $[6,7]$. In recent years, adaptive decoupling theory, fuzzy decoupling theory and neural network decoupling theory have also made great achievements [8-11]. But there are some difficulties when these schemes are applied in practical applications; for example, the decoupling control system based on classical control theory often leads to a physically unrealizable problem, while the decoupling control system based on the modern control theory often leads to complex calculations and its realization is very difficult. As a branch of exact linearization, the input-output linearization is an effective way to decouple MIMO systems [12]. It could be achieved by exact inputoutput transformation and feedback, and any higher-order nonlinear terms are not neglected. Additionally, it could be employed to stabilize systems in a large scale. What's more, it could avoid complicated calculations in dealing with the tracking problem for nonlinear MIMO systems, and it is easy to achieve.

As is well known, optimal control is one of the most important branches in modern control theory and LQRTC has been used and developed well in linear MIMO systems. However, there would be several problems in applying LQRTC to uncertain nonlinear systems. The optimal LQRTC for nonlinear systems often leads to a nonlinear two-point boundary-value (TPBV) problem and the analytical solution generally does not exist except in some simple cases [13]. Additionally, the optimal controller design is usually based on the precise mathematical models. If the system is subject to 
some uncertainties, such as parameter variations, unmodeled dynamics, and external disturbances, the performance criterion which is optimized based on the nominal system would deviate from the optimal value or even the system becomes unstable.

Sliding-mode control (SMC) is an effective robust control approach for uncertain nonlinear systems [14, 15]. Its outstanding advantage is that the sliding motion exhibits complete robustness to system uncertainties [16, 17]. However, during the reaching phase, the SMC system is sensitive to uncertainties. Therefore, various methods have been suggested by minimizing or even removing the reaching phase, such as time-varying sliding mode and integral sliding mode (ISM). Time-varying sliding-mode surfaces that can remove the reaching phase were studied in [18] for the SISO system. And for a class of uncertain MIMO nonlinear systems, three types of time-varying sliding-mode control were proposed in [19]. Another effective method to remove the reaching phase and obtain a global robustness is the ISM, which was proposed by Lee [20] for linear systems. Recently, the ISM control research has obtained many results, for example, the optimal and robust control for linear state-delay systems was proposed in [21], the LMI-based ISM control of mismatched uncertain systems was considered in [22]. Compared with the time-varying sliding mode, the ISM is simpler and easier to implement, especially for MIMO systems. How to make an optimal controller or tracking controller have the global robustness of ISM is a valuable subject. For the optimal control problem, [20] studied the problem for linear systems. Reference [23] proposed a higher order sliding-mode control methodology based on ISM for a class of nonlinear SISO systems. Reference [24] presented an ISM surface for a class of nonlinear uncertain systems based on the exact linearization. Reference [25] studied the global robust optimal slidingmode control based on ISM for class of MIMO nonlinear systems, with the nonlinear LQR problem solved by the sensitivity approach. But with system-order increasing, the complexity for calculating optimal solution increases rapidly. For the optimal tracking control problem, [26] studied the optimal sliding-mode output tracking control for linear uncertain systems with the reference signal given by a linear time-varying exosystem. Reference [27] proposed an optimal output tracking controller for nonlinear systems based on successive approximation approach, without uncertainties considered. Reference [28] studied the optimal sliding-mode control by combining ISM with optimal control and applied it to quaternion-based spacecraft attitude tracking maneuvres with external disturbances and an uncertainty inertia matrix. The control Lyapunov function (CLF) approach and Lyapunov optimizing control (LOC) methods were used to solve the nonlinear optimal control problems, respectively, and the desired reference was given by some known trajectories.

The optimal tracking problem for nonlinear MIMO systems with reference signals generated by a time-varying exosystem is more challenging because of the complexity of nonlinear, the difficulty of the optimal solution, the inevitability of uncertainties, the coupling problem, and so on.
In this paper, the input-output linearization is employed to linearize and decouple the original MIMO system. Based on the decoupled system and the exosystem, an error equation is constructed. Therefore, the optimal tracking problem of original system is transferred into an optimal state regulation problem about the linear error system, and the TPBV problem is avoided. Based on optimal control law, an ISM surface is constructed, which can remove the reaching phase of SMC and guarantee the global sliding mode. To reduce chattering, the reaching law is used to design the optimal sliding-mode tracking control law. As a result, not only the optimal performance can be obtained but also the global robustness to uncertainties is guaranteed. The proposed algorithm is applied to a two-link robot system, and simulation results show its effectiveness.

\section{Problem Formulation}

Consider a class of uncertain affine nonlinear MIMO systems as follows:

$$
\begin{gathered}
\dot{x}=f(x)+\Delta f(x)+G(x) u+d(x, t), \\
y=h(x),
\end{gathered}
$$

where $x \in R^{n}$ is the system state vector, $u \in R^{m}$ is the control input vector, and $y \in R^{m}$ is the system output vector. $G(x)=\left[g_{1}(x), \ldots, g_{m}(x)\right], f(x)$, and $g_{i}(x)$ are sufficiently smooth vector fields on a domain $D \subset R^{n} . h(x)$ is a measured sufficiently smooth output function vector and $h(x)=$ $\left[h_{1}, \ldots, h_{m}\right]^{\mathrm{T}} . \Delta f(x)$ and $d(x, t)$ are unknown function vectors representing the system uncertainties, including system parameter variations, unmodeled dynamics, and external disturbances.

The reference signal $\tilde{y}(t)$ is given by the following exosystem:

$$
\begin{gathered}
\dot{z}(t)=F(t) z(t), \quad z\left(t_{0}\right)=z_{0}, \\
\tilde{y}(t)=H(t) z(t),
\end{gathered}
$$

where $z \in R^{p}$ is the state vector and $\tilde{y} \in R^{m}$ is the output vector. $F(t)$ and $H(t)$ are time-varying matrices with appropriate dimensions. Suppose that the pair $[F(t), H(t)]$ is observable.

Our objective is to design a ROSMTC so that the output $y$ of system (1) can track the exosystem's output $\tilde{y}$ asymptotically, some given performance criterion is minimized and the system can exhibit global robustness to uncertainties.

Ignoring the uncertainties, the nominal system of uncertain affine nonlinear system (1) is

$$
\begin{gathered}
\dot{x}=f(x)+G(x) u, \\
y=h(x) .
\end{gathered}
$$

Assumption 1. Equation (3) has the relative degree vector $\left\{r_{1}, \ldots, r_{m}\right\}$ and $r=r_{1}+\cdots+r_{m}=n$.

Assumption 2. The reference trajectory $\tilde{y}(t)$ and its derivations $\widetilde{y}^{(i)}(t)(i=1, \ldots, n)$ can be obtained online, and they are bounded to all $t \geq 0$. 
The nominal system (3) is a coupling nonlinear MIMO system. In the next part, exact linearization will be employed to transform the nonlinear system into a linear one and achieve decoupling between the inputs and outputs. Furthermore, the original tracking problem will be transformed into a robust optimal regulation problem for linear system.

\section{Input-Output Linearization and Problem Transformation}

Considering system (3) and differentiating $y=h(x)$, we have

$$
\begin{gathered}
y_{i}^{(k)}=L_{f}^{k} h_{i}(x), \quad 0 \leq k \leq r_{i}-1, \\
y_{i}^{\left(r_{i}\right)}=L_{f}^{r_{i}} h_{i}(x)+\sum_{j=1}^{m} L_{g_{j}} L_{f}^{r_{i}-1} h_{i}(x) u_{j} .
\end{gathered}
$$

Define

$$
M(x)=\left[\begin{array}{ccc}
L_{g_{1}} L_{f}^{r_{1}-1} h_{1}(x) & \cdots & L_{g_{m}} L_{f}^{r_{1}-1} h_{1}(x) \\
\vdots & \cdots & \vdots \\
L_{g_{1}} L_{f}^{r_{m}-1} h_{m}(x) & \cdots & L_{g_{m}} L_{f}^{r_{m}-1} h_{m}(x)
\end{array}\right] .
$$

And $M(x)$ is nonsingular in some domain for all $x \in X_{0}$. According to Assumption 1, (4) can be written as

$$
\left[\begin{array}{c}
y_{1}^{\left(r_{1}\right)} \\
\vdots \\
y_{m}^{\left(r_{m}\right)}
\end{array}\right]=\left[\begin{array}{c}
L_{f}^{r_{1}} h_{1}(x) \\
\vdots \\
L_{f}^{r_{m}} h_{m}(x)
\end{array}\right]+M(x)\left[\begin{array}{c}
u_{1} \\
\vdots \\
u_{m}
\end{array}\right] .
$$

Choose the control law in the form of

$$
u=-M^{-1}(x)\left[\begin{array}{c}
L_{f}^{r_{1}} h_{1}(x) \\
\vdots \\
L_{f}^{r_{m}} h_{m}(x)
\end{array}\right]+M^{-1}(x) v ;
$$

then the input-output dynamic equation can be described as

$$
\left[\begin{array}{c}
y_{1}^{\left(r_{1}\right)} \\
\vdots \\
y_{m}^{\left(r_{m}\right)}
\end{array}\right]=\left[\begin{array}{c}
v_{1} \\
\vdots \\
v_{m}
\end{array}\right]
$$

As can be seen, the output $y_{i}=h_{i}(x)$ is only related to the input $v_{i}$, which means the input-output decoupling has been realized. Noting that the relative degree of system (1) is $r=r_{1}+\cdots+r_{m}=n$, so the decoupling process is equivalent to the input-output linearization process. In the following part, the results above will be applied to uncertain affine nonlinear system (1) to structure a tracking error equation. have

Considering system (1) and differentiating $y=h(x)$, we

$$
\begin{aligned}
y_{i}^{(k)} & =L_{f}^{k} h_{i}(x), \quad 0 \leq k \leq r_{i}-1, \\
y_{i}^{\left(r_{i}\right)}= & L_{f}^{r_{i}} h_{i}(x)+L_{\Delta f} L_{f}^{r_{i}-1} h_{i}(x) \\
& +\sum_{j=1}^{m} L_{g_{j}} L_{f}^{r_{i}-1} h_{i}(x) u_{j}+L_{d} L_{f}^{r_{i}-1} h_{i} .
\end{aligned}
$$

Define $\eta_{i}^{j}=L_{f}^{j} h_{i}(x), i=1, \ldots, m$, and $j=0,1, \ldots, \gamma_{i-1}$ and choose the following nonlinear state transformation:

$$
\xi=\left[\eta_{1}^{0}, \ldots, \eta_{1}^{r_{1}-1}, \ldots, \eta_{m}^{0}, \ldots, \eta_{m}^{r_{m}-1}\right]^{\mathrm{T}} .
$$

Define the tracking error as

$$
\begin{aligned}
e= & {\left[h_{1}(x)-\tilde{y}_{1}, \ldots, L_{f}^{\left(r_{1}-1\right)} h_{1}(x)-\widetilde{y}_{1}^{\left(r_{1}-1\right)}, \ldots,\right.} \\
& \left.h_{m}(x)-\tilde{y}_{m}, \ldots, L_{f}^{\left(r_{m}-1\right)} h_{m}(x)-\widetilde{y}_{m}^{\left(r_{m}-1\right)}\right]^{\mathrm{T}} \\
= & {\left[\xi_{1}-y_{1}, \ldots, L_{f}^{\left(r_{1}-1\right)} h_{1}(x)-\widetilde{y}_{1}^{\left(r_{1}-1\right)}, \ldots,\right.} \\
& \left.h_{m}(x)-\tilde{y}_{m}, \ldots, L_{f}^{\left(r_{m}-1\right)} h_{m}(x)-\widetilde{y}_{m}^{\left(r_{m}-1\right)}\right]^{\mathrm{T}} \\
= & {\left[e_{1}, \ldots, e_{1}^{\left(r_{1}-1\right)}, \ldots, e_{m}, \ldots, e_{m}^{\left(r_{m}-1\right)}\right]^{\mathrm{T}} . }
\end{aligned}
$$

Define $\widetilde{Y}=\left[\widetilde{y}_{1}^{r_{1}}, \ldots, \widetilde{y}_{m}^{r_{m}}\right]^{\mathrm{T}} \in R^{m}$ and choose the control law in the form of

$$
u=M^{-1}(x)[-b(x)+\widetilde{Y}+v],
$$

where $b(x)=\left[\begin{array}{lll}L_{f}^{\gamma_{1}} h_{1} & \cdots & L_{f}^{\gamma_{m}} h_{m}\end{array}\right]^{\mathrm{T}}$.

So the tracking error equation can be written as

$$
\dot{e}=\bar{A} e+\Delta \bar{A}+\bar{B} v+\Delta \bar{B},
$$

where $e \in R^{n}$ is the system tracking error vector and $v \in R^{m}$ is a new control input of the transformed system. $\bar{A}, \Delta \bar{A}$, $\bar{B}$, and $\Delta \bar{B}$ are corresponding constant matrices and defined, respectively, as follows:

$$
\begin{aligned}
& \bar{A}=\operatorname{diag}\left(\bar{A}_{1}, \ldots, \bar{A}_{m}\right), \\
& \bar{B}=\operatorname{diag}\left(\bar{B}_{1}, \ldots, \bar{B}_{m}\right), \\
& \Delta \bar{A}=\left[\Delta \bar{A}_{1}, \ldots, \Delta \bar{A}_{m}\right]^{\mathrm{T}}, \\
& \Delta \bar{B}=\left[\Delta \bar{B}_{1}, \ldots, \Delta \bar{B}_{m}\right]^{\mathrm{T}}, \\
& \bar{A}_{i}=\left[\begin{array}{ccccc}
0 & 1 & 0 & \cdots & 0 \\
0 & 0 & 1 & \cdots & 0 \\
\vdots & \vdots & \vdots & & \vdots \\
0 & 0 & 0 & \cdots & 1 \\
0 & 0 & 0 & 0 & 0
\end{array}\right]_{r_{i} \times r_{i}}, \quad \bar{B}_{i}=\left[\begin{array}{c}
0 \\
0 \\
\vdots \\
0 \\
1
\end{array}\right]_{r_{i} \times 1} \text {, } \\
& \Delta \bar{A}_{i}=\left[\begin{array}{c}
0 \\
0 \\
\vdots \\
0 \\
L_{\Delta f} L_{f}^{r_{i}-1} h_{i}(x)
\end{array}\right]_{r_{i} \times 1}, \\
& \Delta \bar{B}_{i}=\left[\begin{array}{c}
0 \\
0 \\
\vdots \\
0 \\
L_{d} L_{f}^{r_{i}-1} h_{i}(x)
\end{array}\right]_{r_{i} \times 1},
\end{aligned}
$$


where $\Delta \bar{A}$ and $\Delta \bar{B}$ represent uncertainties of the transformed system. Obviously, $\Delta \bar{A}$ and $\Delta \bar{B}$ satisfy the matching conditions; that is, there exist unknown continuous function vectors $\Delta \widetilde{A}\left(\in R^{m}\right)$ and $\Delta \widetilde{B}\left(\in R^{m}\right)$ which satisfy

$$
\Delta \bar{A}=\bar{B} \Delta \widetilde{A}, \quad \Delta \bar{B}=\bar{B} \Delta \widetilde{B} .
$$

Assumption 3. There exist known constants $a_{m}$ and $b_{m}$ such that

$$
\|\Delta \widetilde{A}\|_{1} \leq a_{m}, \quad\|\Delta \widetilde{B}\|_{1} \leq d_{m},
$$

where $\|\cdot\|_{1}$ denotes the 1-norm.

After exact linearization and decoupling, the optimal tracking problem of original system (1) is transferred into a robust optimal regulation problem about the error system (13). In the next part, the ROSMTC will be designed for system (13).

\section{Design of Robust Optimal Sliding-Mode Tracking Controller (ROSMTC)}

4.1. Optimal Tracking Control of Nominal System. Ignoring the uncertainties of system (13), the corresponding nominal system is

$$
\dot{e}(t)=\bar{A} e(t)+\bar{B} v(t) .
$$

For (17), let $v=v_{0}$ and $v_{0}$ can minimize the quadratic performance index as follows:

$$
J_{N}=\frac{1}{2} \int_{0}^{\infty}\left[e^{\mathrm{T}}(t) Q e(t)+v_{0}^{\mathrm{T}}(t) R v_{0}(t)\right] \mathrm{d} t,
$$

where $Q \in R^{n \times n}$ is a symmetric semipositive definite matrix and $R \in R^{m \times m}$ is a positive definite matrix.

According to optimal control theory, the optimal feedback control law can be described as

$$
v_{0}(t)=-R^{-1} \bar{B}^{\mathrm{T}} P e(t),
$$

where $P$ is the solution of matrix Riccati equation as follows:

$$
P \bar{A}+\bar{A}^{\mathrm{T}} P-P \bar{B} R^{-1} \bar{B}^{\mathrm{T}} P+Q=0 .
$$

So the closed-loop system dynamics is

$$
\dot{e}(t)=\left(\bar{A}-\bar{B} R^{-1} \bar{B}^{\mathrm{T}} P\right) e(t) .
$$

According to optimal control theory, the closed-loop system is asymptotically stable. However, if the control law (19) is applied to uncertain system (13), the state trajectory will deviate from the optimal trajectory and even the system will become unstable. Next we will adopt ISM control technique to robustify the optimal control law; to achieve the goal that the state trajectory of uncertain system (13) is the same as that of the optimal trajectory of the nominal system (17).
4.2. The Robust Optimal Sliding Surface. Considering the uncertain system (13), we define an integral sliding surface in the form of

$$
s(e, t)=\bar{G} e(t)-\bar{G} \int_{0}^{t}\left(\bar{A}-\bar{B} R^{-1} \bar{B}^{\mathrm{T}} P\right) e(\tau) \mathrm{d} \tau-\overline{\mathrm{G}} e(0),
$$

where $\bar{G} \in R^{m \times n}$ which satisfies that $\bar{G} \bar{B}$ is nonsingular and $e(0)$ is the initial state vector. Differentiating (22) with respect to $t$ and considering (13), we obtain

$$
\begin{aligned}
\dot{s}(e, t)= & \bar{G} \dot{e}(t)-\bar{G}\left(\bar{A}-\bar{B} R^{-1} \bar{B}^{\mathrm{T}} P\right) e(t) \\
= & \bar{G}[\bar{A} e(t)+\Delta \bar{A}+\bar{B} v(t)+\Delta \bar{B}] \\
& -\bar{G}\left(\bar{A}-\bar{B} R^{-1} \bar{B}^{\mathrm{T}} P\right) e(t) \\
= & \bar{G} \bar{B} v(t)+\bar{G} \Delta \bar{A}+\bar{G} \Delta \bar{B}+\bar{G} \bar{B} R^{-1} \bar{B}^{\mathrm{T}} P e(t) .
\end{aligned}
$$

Let $\dot{s}(t)=0$; then the equivalent control becomes

$$
v_{\mathrm{eq}}(t)=-(\bar{G} \bar{B})^{-1}\left[\bar{G} \Delta A+\bar{G} \Delta \bar{B}+\bar{G} B R^{-1} B^{\mathrm{T}} P e(t)\right] .
$$

Substituting (24) into (13) and considering (15), the slidingmode dynamics is

$$
\begin{aligned}
\dot{e}(t)= & \bar{A} e(t)+\Delta \bar{A}+\bar{B} v_{\mathrm{eq}}(t)+\Delta \bar{B} \\
= & \bar{A} e(t)+\Delta \bar{A}-\bar{B}(\bar{G} \bar{B})^{-1} \\
& \times\left[\bar{G} \Delta \bar{A}+\bar{G} \Delta \bar{B}+\bar{G} \bar{B} R^{-1} \bar{B}^{\mathrm{T}} P e(t)\right]+\Delta \bar{B} \\
= & \bar{A} e(t)+\Delta \bar{A}-\Delta \bar{A}-\Delta \bar{B} \\
& -\bar{G}^{-1} \bar{G} \bar{B} R^{-1} \bar{B}^{\mathrm{T}} P e(t)+\Delta \bar{B} \\
= & \left(\bar{A}-\bar{B} R^{-1} \bar{B}^{\mathrm{T}} P\right) e(t) .
\end{aligned}
$$

Comparing (25) with (21), we can see that the sliding mode of uncertain linear system (13) is the same as optimal dynamics of (17); therefore, the sliding mode is also asymptotically stable, and the sliding motion guarantees the controlled system global robustness to the uncertainties which satisfy the matching condition. We call (22) a global robust optimal sliding surface.

4.3. The Control Law. For uncertain system (13), we propose the control law as follows:

$$
\begin{gathered}
v(t)=v_{c}(t)+v_{d}(t), \\
v_{c}(t)=-R^{-1} \bar{B}^{\mathrm{T}} P e(t), \\
v_{d}(t)=-(\bar{G} \bar{B})^{-1}[k s+\varepsilon \operatorname{sgn}(s)],
\end{gathered}
$$

where $\operatorname{sgn}(s)=\left[\operatorname{sgn}\left(s_{1}\right), \ldots, \operatorname{sgn}\left(s_{m}\right)\right]^{\mathrm{T}}$ and $k$ and $\varepsilon$ are appropriate positive constants, respectively. $v_{c}(t)$, used to stabilize 
and optimize the nominal system, is the continuous part of the control law. $v_{d}(t)$ is the discontinuous part, which provides complete compensation for uncertainties of system (13).

Theorem 4. Consider uncertain system (13) with Assumption 3. Let the input $v(t)$ and the sliding surface be given by (26) and (22), respectively. The control law can force the system trajectories to reach the sliding surface in finite time and maintain it thereafter if $\varepsilon \geq\left(a_{m}+d_{m}\right)\|\bar{G} \bar{B}\|_{1}$.

Proof. Utilizing $V=(1 / 2) s^{\mathrm{T}} s$ as a Lyapunov function candidate and considering Assumption 3, we obtain

$$
\begin{aligned}
& \dot{V}=s^{\mathrm{T}} \dot{\boldsymbol{s}} \\
& =s^{\mathrm{T}}\left[\bar{G} \dot{e}(t)-\bar{G}\left(\bar{A}-\bar{B} R^{-1} \bar{B}^{\mathrm{T}} P\right) e(t)\right] \\
& =s^{\mathrm{T}}\{\bar{G}[\bar{A} e(t)+\Delta \bar{A}+\bar{B} v(t)+\Delta \bar{B}] \\
& \left.-\bar{G}\left(\bar{A}-\bar{B} R^{-1} \bar{B}^{\mathrm{T}} P\right) e(t)\right\} \\
& =s^{\mathrm{T}}\left\{\bar{G} \Delta \bar{A}+\bar{G} \bar{B}\left[-R^{-1} \bar{B}^{\mathrm{T}} P e(t)-(\bar{G} \bar{B})^{-1}\right.\right. \\
& \times(k s+\varepsilon \operatorname{sgn}(s))+\bar{G} \Delta \bar{B}] \\
& \left.-\bar{G} \bar{B} R^{-1} \bar{B}^{\mathrm{T}} P e(t)\right\} \\
& =s^{\mathrm{T}}\{\bar{G} \Delta \bar{A}+\bar{G} \Delta \bar{B}-[k s+\varepsilon \operatorname{sgn}(s)]\} \\
& =-k\|s\|_{2}^{2}-\varepsilon\|s\|_{1}+s^{\mathrm{T}}(\bar{G} \Delta \bar{A}+\bar{G} \Delta \bar{B}),
\end{aligned}
$$

where $\|\cdot\|_{2}$ denotes the 2-norm. As $s^{\mathrm{T}}(\overline{\mathrm{G}} \Delta \bar{A}+\bar{G} \Delta \bar{B})$ is a scalar quantity considering (15) and Assumption 3, we get

$$
\begin{aligned}
s^{\mathrm{T}}(\bar{G} \Delta \bar{A}+\bar{G} \Delta \bar{B}) & \leq\left\|s^{\mathrm{T}}(\bar{G} \Delta \bar{A}+\bar{G} \Delta \bar{B})\right\|_{1} \\
& =\left\|s^{\mathrm{T}}(\bar{G} \bar{B} \Delta \widetilde{A}+\bar{G} \bar{B} \Delta \widetilde{B})\right\|_{1} \\
& \leq\|s\|_{1} \cdot\|\bar{G} \bar{B}\|_{1} \cdot\left(\|\Delta \widetilde{A}\|_{1}+\|\Delta \widetilde{B}\|_{1}\right) \\
& \leq\|s\|_{1} \cdot\|\bar{G} \bar{B}\|_{1} \cdot\left(a_{m}+d_{m}\right) .
\end{aligned}
$$

Thus,

$$
\begin{aligned}
\dot{V}= & s^{\mathrm{T}} \dot{s} \leq-k\|s\|_{2}^{2}-\varepsilon\|s\|_{1} \\
& +\left(a_{m}+d_{m}\right) \cdot\|\bar{G} \bar{B}\|_{1} \cdot\|s\|_{1} .
\end{aligned}
$$

So, if

$$
\varepsilon \geq\left(a_{m}+d_{m}\right)\|\bar{G} \bar{B}\|_{1},
$$

then

$$
\begin{aligned}
\dot{V}= & s^{\mathrm{T}} \dot{\boldsymbol{s}} \leq-k\|s\|_{2}^{2} \\
& -\left[\varepsilon-\left(a_{m}+d_{m}\right)\|\bar{G} \bar{B}\|_{1}\right]\|s\|_{1}<0 .
\end{aligned}
$$

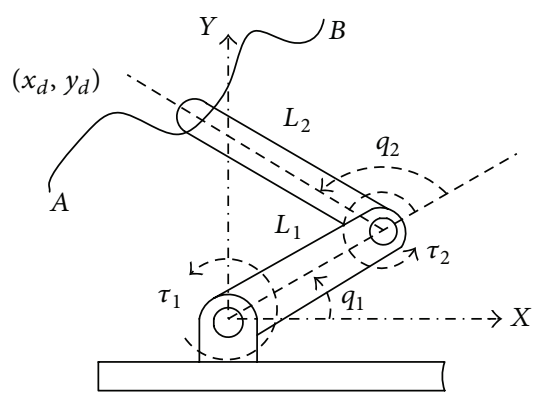

Figure 1: The structure of two-link robot manipulator.

This implies that the trajectories of uncertain system (13) will be globally driven onto the specified sliding surface $s=$ 0 in finite time despite of the uncertainties. The proof is completed.

From (22), we have $s(0)=0$; that is, the initial condition is on the sliding surface. According to Theorem 4, uncertain system (13) achieves global sliding mode with the integral sliding surface (22) and the control law (26). So the system designed is globally robust and optimal.

\section{Application to a Two-Link Robot Manipulator}

Trajectory tracking of multilink robot manipulator has received a great deal of attention in recent years. But it is rather difficult to perform excellent tracking because multijoint robot manipulator is a complex system with high nonlinearity, coupling, and time-varying dynamic behavior. To verify the effectiveness and superiority of the proposed method, we apply it to a two-link robot manipulator in comparison with conventional LQRTC.

Consider a two-link robot manipulator shown in Figure 1.

In this figure, $L_{1}$ and $L_{2}$ denote the machine arms, $\tau_{1}$ and $\tau_{2}$ denote the driving torque, $q_{1}$ and $q_{2}$ present the angular displacement of the two joints, respectively, and $A-B$ is the tracking trajectory described by $\left(x_{d}, y_{d}\right)$. The dynamic equation is given by [29]

$$
M(q) \ddot{q}+C(q, \dot{q}) \dot{q}+G(q)+\mathrm{d}(t)=\tau,
$$

where $q=\left[\begin{array}{ll}q_{1} & q_{2}\end{array}\right]^{\mathrm{T}}$ is the joint-displacement vector, $\tau=$ $\left[\tau_{1}, \tau_{2}\right]^{\mathrm{T}}$ is the applied joint-torque vector, and $\mathrm{d}(t)$ represents system uncertainties. $M(q), C(q, \dot{q}), G(q)$, and $g$ are defined as follows:

$$
\begin{gathered}
M(q)=\left[\begin{array}{cc}
0.1+0.01 \cos \left(q_{2}\right) & 0.01 \sin \left(q_{2}\right) \\
0.01 \sin \left(q_{2}\right) & 0.1
\end{array}\right], \\
C(q, \dot{q})=\left[\begin{array}{cc}
-0.005 \sin \left(q_{2}\right) \dot{q}_{2} & 0.005 \cos \left(q_{2}\right) \dot{q}_{2} \\
0.005 \cos \left(q_{2}\right) \dot{q}_{2} & 0
\end{array}\right],
\end{gathered}
$$




$$
\begin{gathered}
G(q)=\left[\begin{array}{ll}
0.01 g \cos \left(q_{1}+q_{2}\right) \\
0.01 g \cos \left(q_{1}+q_{2}\right)
\end{array}\right], \quad g=9.8, \\
\mathrm{~d}(t)= \begin{cases}{\left[\begin{array}{l}
0 \\
0
\end{array}\right],} & t<5 \mathrm{~s}, \\
{\left[\begin{array}{c}
\sin (0.5 \pi t) \\
10 \sin (3 \pi t)
\end{array}\right],} & t \geq 5 \mathrm{~s} .\end{cases}
\end{gathered}
$$

Suppose the reference signal is given by the following exosystem:

$$
\begin{gathered}
\dot{z}(t)=\left[\begin{array}{cc}
-1 & 4 \\
-5 & -1
\end{array}\right] z(t), \\
\tilde{y}(t)=\left[\begin{array}{ll}
1 & 1 \\
5 & 5
\end{array}\right] z(t) .
\end{gathered}
$$

Our objective is to design an robust optimal tracking controller, such that the $q_{1}, q_{2}, \dot{q}_{1}$, and $\dot{q}_{2}$ can track $\tilde{y}_{1}, \tilde{y}_{2}, \dot{\tilde{y}}_{1}$, and $\dot{\tilde{y}}_{2}$, respectively. Therefore, a certain given performance criterion can be minimized and the system can exhibit robustness to uncertainties.

Choose a state vector as follows:

$$
\xi=\left[\begin{array}{llll}
\xi_{1} & \xi_{2} & \xi_{3} & \xi_{4}
\end{array}\right]^{\mathrm{T}}=\left[\begin{array}{llll}
q_{1} & \dot{q}_{1} & q_{2} & \dot{q}_{2}
\end{array}\right]^{\mathrm{T}} .
$$

Define $e=\xi-\tilde{y}=\left[q_{1}-\tilde{y}_{1}, \dot{q}_{1}-\dot{\tilde{y}}_{1}, q_{2}-\tilde{y}_{2}, q_{2}-\dot{\tilde{y}}_{2}\right]^{\mathrm{T}}$ and let the control law

$$
\tau=M(q)\left[\begin{array}{c}
v_{1}+\ddot{\tilde{y}}_{1} \\
v_{2}+\ddot{\tilde{y}}_{2}
\end{array}\right]+C(q, \dot{q}) \dot{q}+G(q) .
$$

So the error state dynamic of the robot manipulator can be written as

$$
\begin{aligned}
\dot{e}= & {\left[\begin{array}{llll}
0 & 1 & 0 & 0 \\
0 & 0 & 0 & 0 \\
0 & 0 & 0 & 1 \\
0 & 0 & 0 & 0
\end{array}\right] e } \\
& +\left[\begin{array}{ll}
0 & 0 \\
1 & 0 \\
0 & 0 \\
0 & 1
\end{array}\right] v-\left[\begin{array}{ll}
0 & 0 \\
1 & 1 \\
0 & 1 \\
1 & 1
\end{array}\right] M^{-1}(q) \mathrm{d}(t) .
\end{aligned}
$$

The quadratic performance index is chosen as (18) and the weighting matrices are

$$
\begin{gathered}
Q=\left[\begin{array}{cccc}
100 & 1.5 & 1.5 & 1.5 \\
1.5 & 1 & 1 & 1 \\
1.5 & 1 & 1 & 1 \\
1.5 & 1 & 1 & 1
\end{array}\right], \\
R=\left[\begin{array}{ll}
0.02 & 0.01 \\
0.01 & 0.01
\end{array}\right] .
\end{gathered}
$$

In order to show the efficiency and the advantage of the proposed approach, a conventional optimal LQRTC for the nominal system and a ROSMTC for the uncertain system

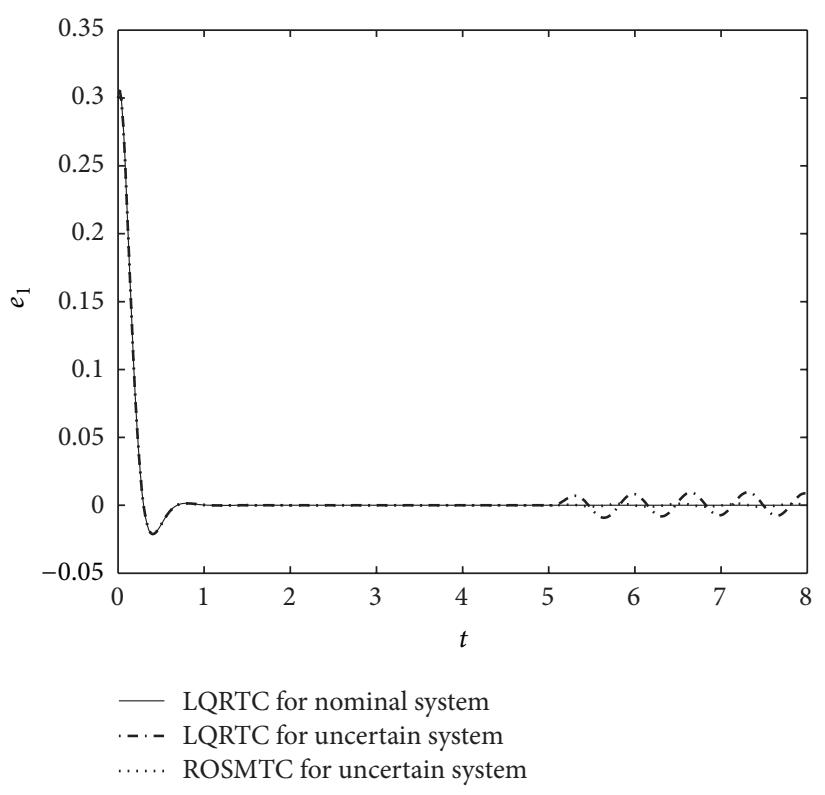

Figure 2: The tracking error $e_{1}(t)$ of position $q_{1}$ for link 1 .

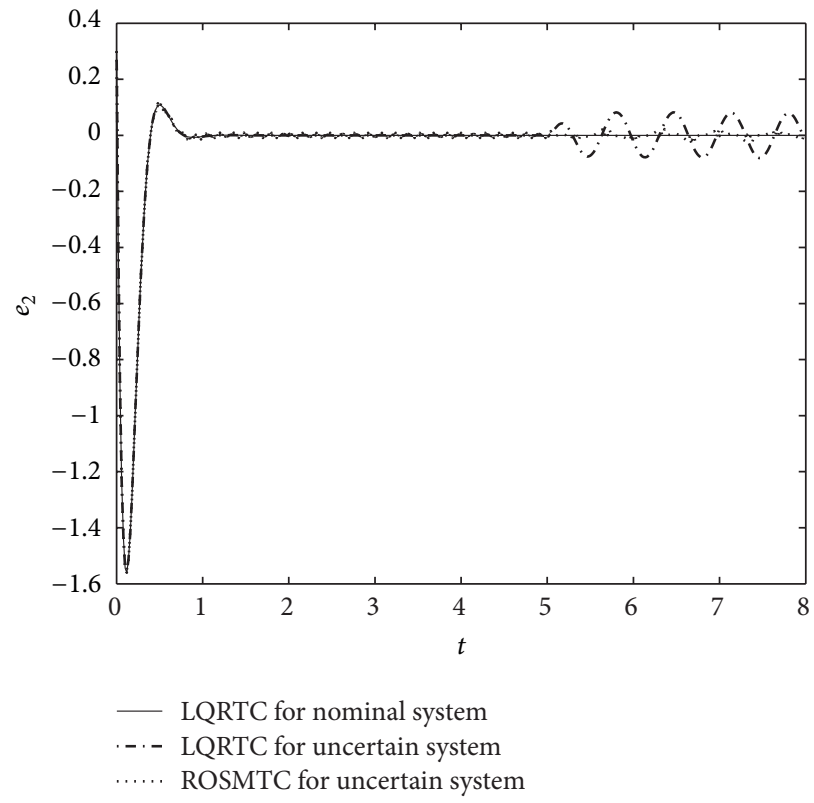

FIGURE 3: The tracking error $e_{2}(t)$ of velocity $\dot{q}_{1}$ for link 1 .

are designed, respectively. For ROSMTC, the sliding-mode surface is chosen in the form of (22) and the control law is chosen in the form of (26) with the designed parameters as follows:

$$
\bar{G}=\left[\begin{array}{cccc}
0 & 1.5 & 0 & 0 \\
0 & 0 & 0 & 1.5
\end{array}\right], \quad k=5, \quad \varepsilon=1.6
$$

With the initial state vectors $\left[\begin{array}{llll}q_{10} & \dot{q}_{10} & q_{20} & \dot{q}_{20}\end{array}\right]^{\mathrm{T}}=$ $\left[\begin{array}{llll}0.5 & 0 & 0.5 & 0\end{array}\right]^{\mathrm{T}}$ and $\left[\begin{array}{ll}z_{10} & z_{20}\end{array}\right]^{\mathrm{T}}=\left[\begin{array}{ll}0.1 & 0.1\end{array}\right]^{\mathrm{T}}$, the simulation results are shown in Figures 2-9. 


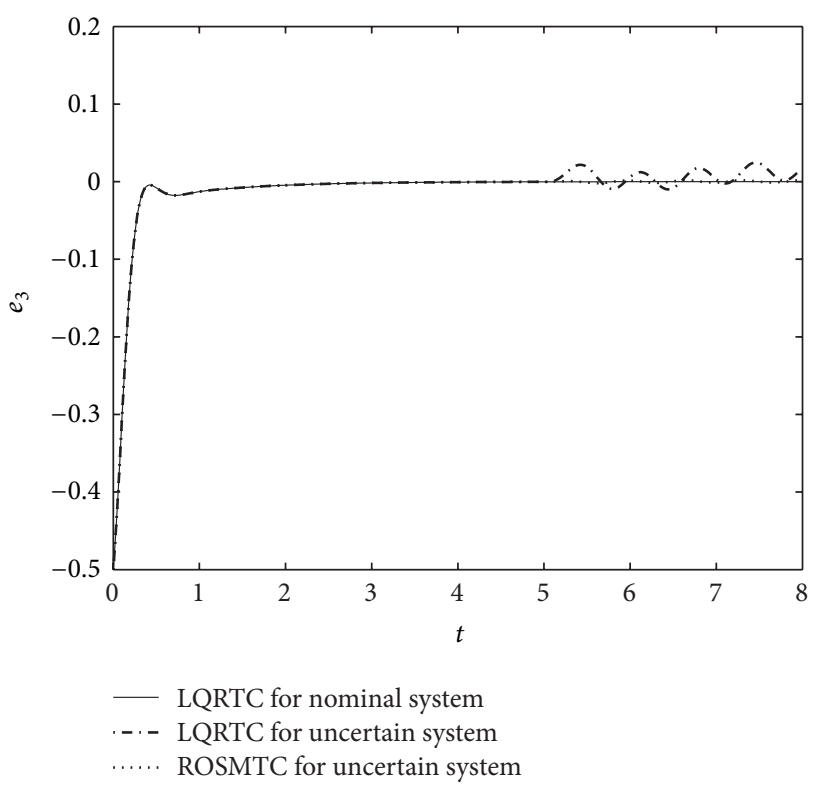

FIGURE 4: The tracking error $e_{3}(t)$ of position $q_{2}$ for link 2 .

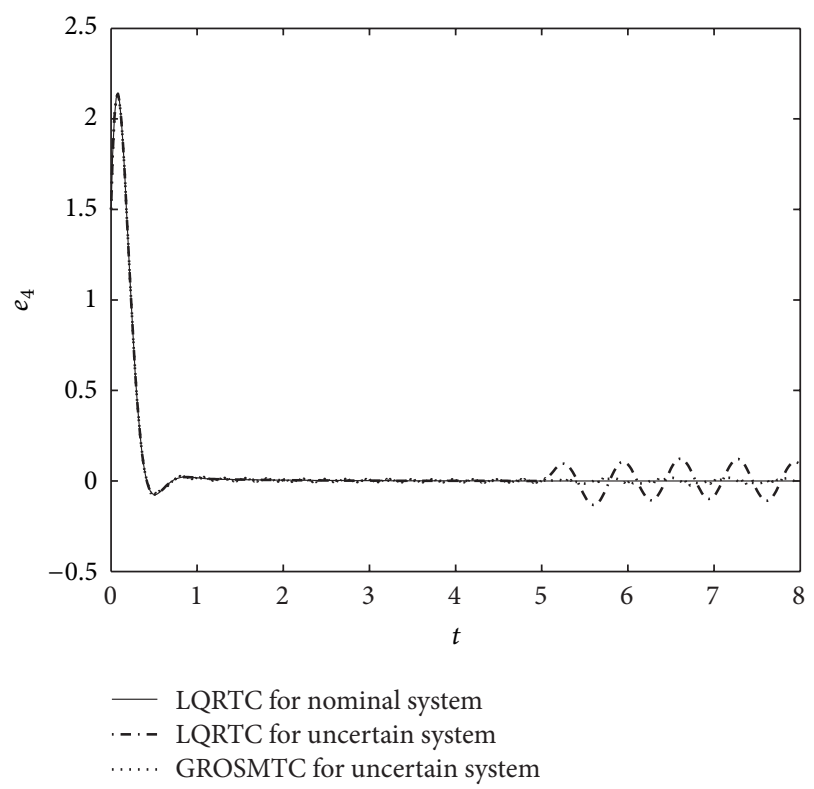

FIgURE 5: The tracking error $e_{4}(t)$ of velocity $\dot{q}_{2}$ for link 2 .

Figures 2, 3, 4, and 5 show the system responses in the following three cases: LQRTC for system (32) with $\mathrm{d}(t)=0$, LQRTC for system (32) with the given $\mathrm{d}(t)$, and ROSMTC for system (32) with the given $\mathrm{d}(t)$. It can be seen from Figure 2 that when the system is subject to uncertainties, the response of the system with LQRTC deviates from the optimal trajectory, however, the response of the system with ROSMTC is almost the same as that of the nominal system with LQRTC.

The output tracking curves are shown in Figures 6, 7, 8 , and 9. It can be seen that, without external disturbance, the controlled system could track the exosystem output by

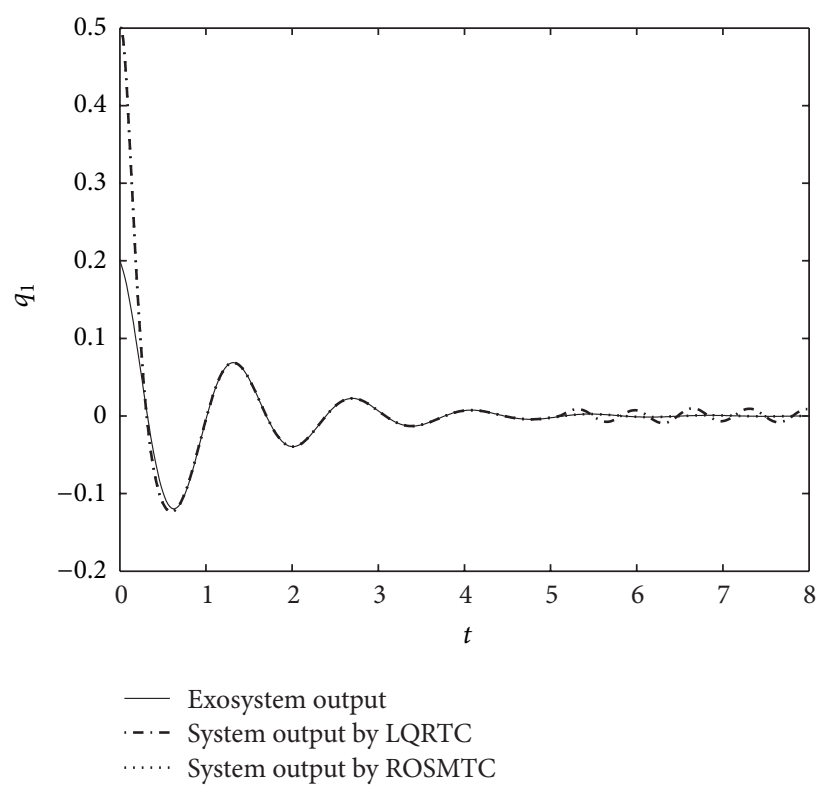

FIgURE 6: The tracking response curve of the position $q_{1}$ for link 1 .

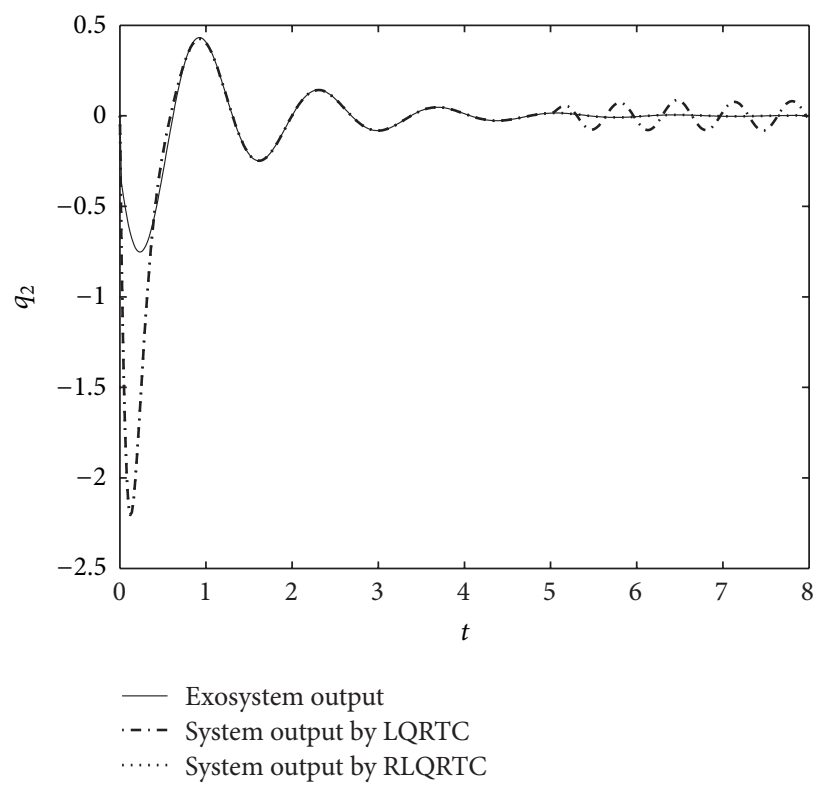

FIGURE 7: The tracking response curve of the velocity $\dot{q}_{1}$ for link 1 .

both controllers at about $t=0.7 \mathrm{~s}$. However, when the external disturbance influences the system at $t=5 \mathrm{~s}$, the output trajectory of LQRTC deviates from the desired trajectory while the tracking performance of ROSMTC is almost not affected. Thus, the ROSMTC provides better features than conventional LQRTC in terms of robustness to system uncertainties.

\section{Conclusions}

A robust optimal tracking control for a class of affine nonlinear MIMO systems with the reference signal given by an 


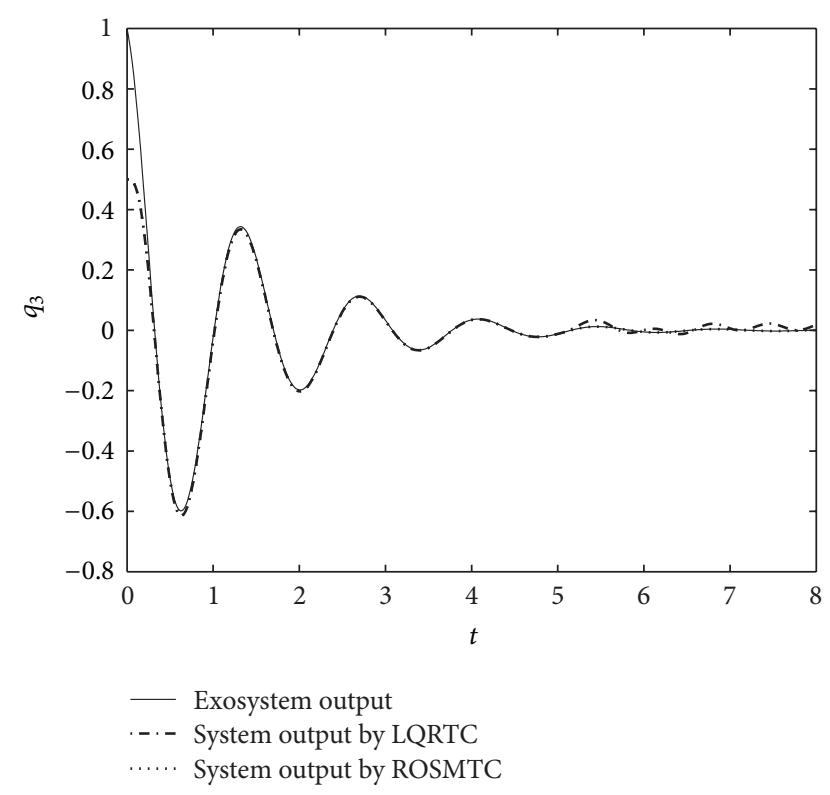

FIGURE 8: The tracking response curve of the position $q_{2}$ for link 2 .

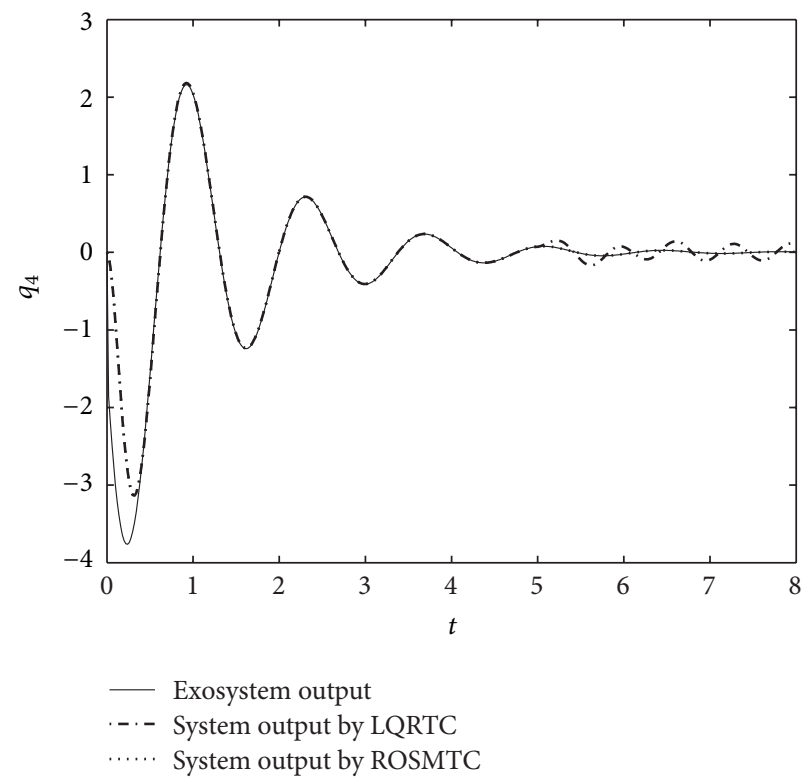

FIGURE 9: The tracking response curve of the velocity $\dot{q}_{2}$ for link 2 .

exosystem has been studied. A linear tracking error equation, with the input and output decoupled, has been established based on the input-output linearization technique. And the nonlinear optimal tracking problem was transformed into a linear LQRTC problem. Moreover, SMC has been used to robustify the LQRTC and a global ROSMTC was realized. That is, the tracking dynamics exhibits global robustness to the uncertainties and the given quadratic performance index can be minimized. The proposed controller was applied to a two-link robot system and simulation results show that good tracking performance can be achieved and global robustness to the uncertainties can be achieved.

\section{Acknowledgments}

This work was supported in part by the National Natural Science Foundation of China (Grant nos. 60940018 and 61104004), and the Shandong Natural Science Foundation (Grant no. ZR2011FQ006).

\section{References}

[1] Y.-J. Pan, H. J. Marquez, and T. Chen, "Robust tracking control for a class of MIMO nonlinear systems with measurable output feedback," International Journal of Robust and Nonlinear Control, vol. 18, no. 1, pp. 69-87, 2008.

[2] Y.-C. Chang, "Robust tracking control for nonlinear MIMO systems via fuzzy approaches," Automatica, vol. 36, no. 10, pp. 1535-1545, 2000.

[3] T.-C. Lee and Z.-P. Jiang, "New cascade approach for global $\kappa$-exponential tracking of underactuated ships," IEEE Transactions on Automatic Control, vol. 49, no. 12, pp. 2297-2303, 2004.

[4] M. Shen and H. Guang, " $H_{2}$ state feedback controller design for continuous Markov jump linear systems with partly known information," International Journal of Systems Science. Principles and Applications of Systems and Integration, vol. 43, no. 4, pp. 786-796, 2012.

[5] F. N. Koumboulis and M. G. Skarpetis, "Output feedback decoupling of linear systems with nonlinear uncertain structure," Journal of the Franklin Institute B, vol. 333, no. 4, pp. 625-629, 1996.

[6] J. L. Neves, B. Heitmann, and N. Khaneja, "Heteronuclear decoupling by optimal tracking," Journal of Magnetic Resonance, vol. 201, no. 11, pp. 7-17, 2009.

[7] Y. S. Zhou and X. R. Zhu, "Decoupling control of the rotor crosscoupling voltage for doubly-fed induction generator based on multiple Degree-Freedom Internal Model Control," in Proceedings of Control and Decision Conference (CCDC '10), pp. 31583163, Yantai, China, 2010.

[8] W. X. Shi, M. Zhang, W. C. Guo, and L. Guo, "Stable adaptive fuzzy control for MIMO nonlinear systems," Computers \& Mathematics with Applications, vol. 62, no. 7, pp. 2843-2853, 2011.

[9] Y.-C. Chang, "An adaptive $H^{\infty}$ tracking control for a class of nonlinear multiple-input-multiple-output (MIMO) systems," IEEE Transactions on Automatic Control, vol. 46, no. 9, pp. 14321437, 2001.

[10] L. Salim and B. M. Seghir, "Indirect fuzzy adaptive control of a class of SISO nonlinear systems," The Arabian Journal for Science and Engineering B, vol. 31, no. 1, pp. 61-74, 2006.

[11] Q. Yang, Z. Yang, and Y. Sun, "Universal neural network control of MIMO uncertain nonlinear systems," IEEE Transactions on Neural Networks, vol. 23, pp. 1162-1169, 2012.

[12] D. X. Gao and Z. Q. Sun, "Fuzzy tracking control design for hypersonic vehicles via T-S model," Science China. Information Sciences, vol. 54, no. 3, pp. 521-528, 2011.

[13] G.-Y. Tang, "Suboptimal control for nonlinear systems: a successive approximation approach," Systems \& Control Letters, vol. 54, no. 5, pp. 429-434, 2005.

[14] J. F. Guo and C. C. Gao, "Variable structure control of linear time-delay uncertain singular system," International Journal of Systems, Control and Communications, vol. 1, no. 3, pp. 364-374, 2009. 
[15] S. Bououden, M. Chadli, and H. R. Karimi, "Fuzzy sliding mode controller design using Takagi-Sugeno modelled nonlinear systems," Mathematical Problems in Engineering, vol. 2013, Article ID 734094, 7 pages, 2013.

[16] Y. Q. Xia, J. Chen, G.-P. Liu, L. Wang, and D. Rees, "Robust adaptive sliding mode control for uncertain time-delay systems," International Journal of Adaptive Control and Signal Processing, vol. 23, no. 9, pp. 863-881, 2009.

[17] S.-B. Choi, D.-W. Park, and S. Jayasuriya, "A time-varying sliding surface for fast and robust tracking control of secondorder uncertain systems," Automatica, vol. 30, no. 5, pp. 899904, 1994.

[18] A. Bartoszewicz, "A comment on a time-varying sliding surface for fast and robust tracking control of second-order uncertain systems," Automatica, vol. 31, no. 12, pp. 1893-1895, 1995.

[19] Y. Q. Jin, X. D. Liu, Q. Wei, and C. Hou, “Time-varying sliding mode control for a class of uncertain MIMO nonlinear system subject to control input constraint," Science China. Information Sciences, vol. 53, no. 1, pp. 89-100, 2010.

[20] J.-H. Lee, "Highly robust position control of BLDDSM using an improved integral variable structure system," Automatica, vol. 42, no. 6, pp. 929-935, 2006.

[21] M. Basin, J. Rodriguez-Gonzalez, and L. Fridman, "Optimal and robust control for linear state-delay systems," Journal of the Franklin Institute. Engineering and Applied Mathematics, vol. 344, no. 6, pp. 830-845, 2007.

[22] H. H. Choi, "LMI-based sliding surface design for integral sliding mode control of mismatched uncertain systems," IEEE Transactions on Automatic Control, vol. 52, no. 4, pp. 736-742, 2007.

[23] S. Laghrouche, F. Plestan, and A. Glumineau, "Higher-order sliding mode control based on integral sliding mode," Automatica, vol. 43, no. 3, pp. 531-537, 2007.

[24] H. P. Pang and X. Chen, "Global robust optimal sliding mode control for uncertain affine nonlinear systems," Journal of Systems Engineering and Electronics, vol. 20, no. 4, pp. 838-843, 2009.

[25] H. P. Pang and G. Y. Tang, "Global robust optimal sliding mode control for a class of nonlinear systems with uncertainties," ICIC Express Letters B, vol. 4, no. 6, pp. 2501-2508, 2010.

[26] H. P. Pang, P. Zhang, and N. N. Sun, "Optimal sliding mode output tracking control for linear systems with uncertainties based on observers," Proceedings of the 24th Chinese Control and Decision Conference, pp. 2432-2436, 2012.

[27] G. Y. Tang and M. Q. Fan, "Series-based approximate approach of optimal tracking control for nonlinear systems with timedelay," Progress in Natural Science, vol. 18, no. 12, pp. 1571-1576, 2008.

[28] C. Pukdeboon and A. S. I. Zinober, "Control Lyapunov function optimal sliding mode controllers for attitude tracking of spacecraft," Journal of the Franklin Institute. Engineering and Applied Mathematics, vol. 349, no. 2, pp. 456-475, 2012.

[29] L. Salim and B. M. Seghir, "Global sliding-mode control with generalized sliding dynamics," Asian Journal of Control, vol. 11, no. 4, pp. 449-456, 2009. 


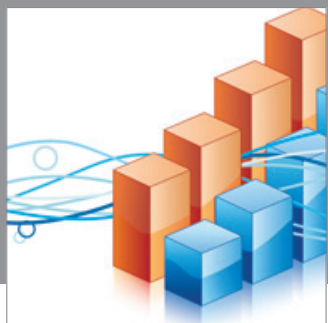

Advances in

Operations Research

mansans

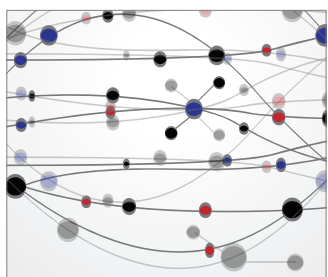

The Scientific World Journal
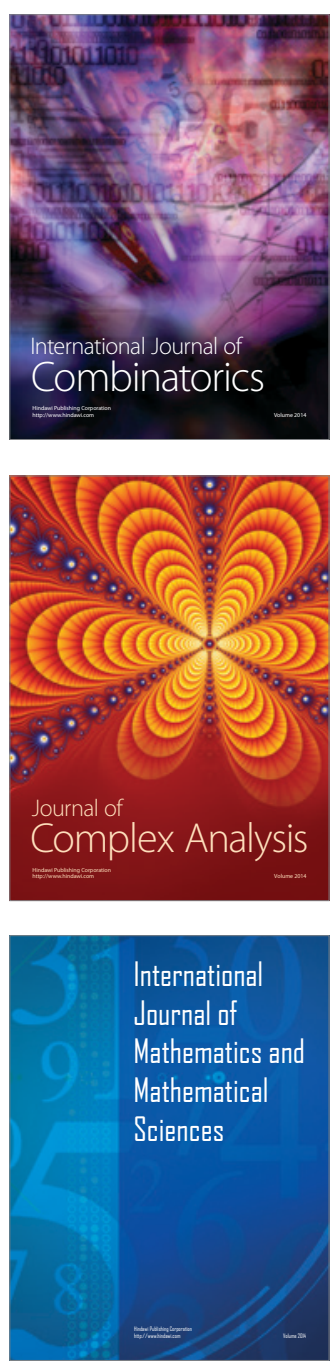
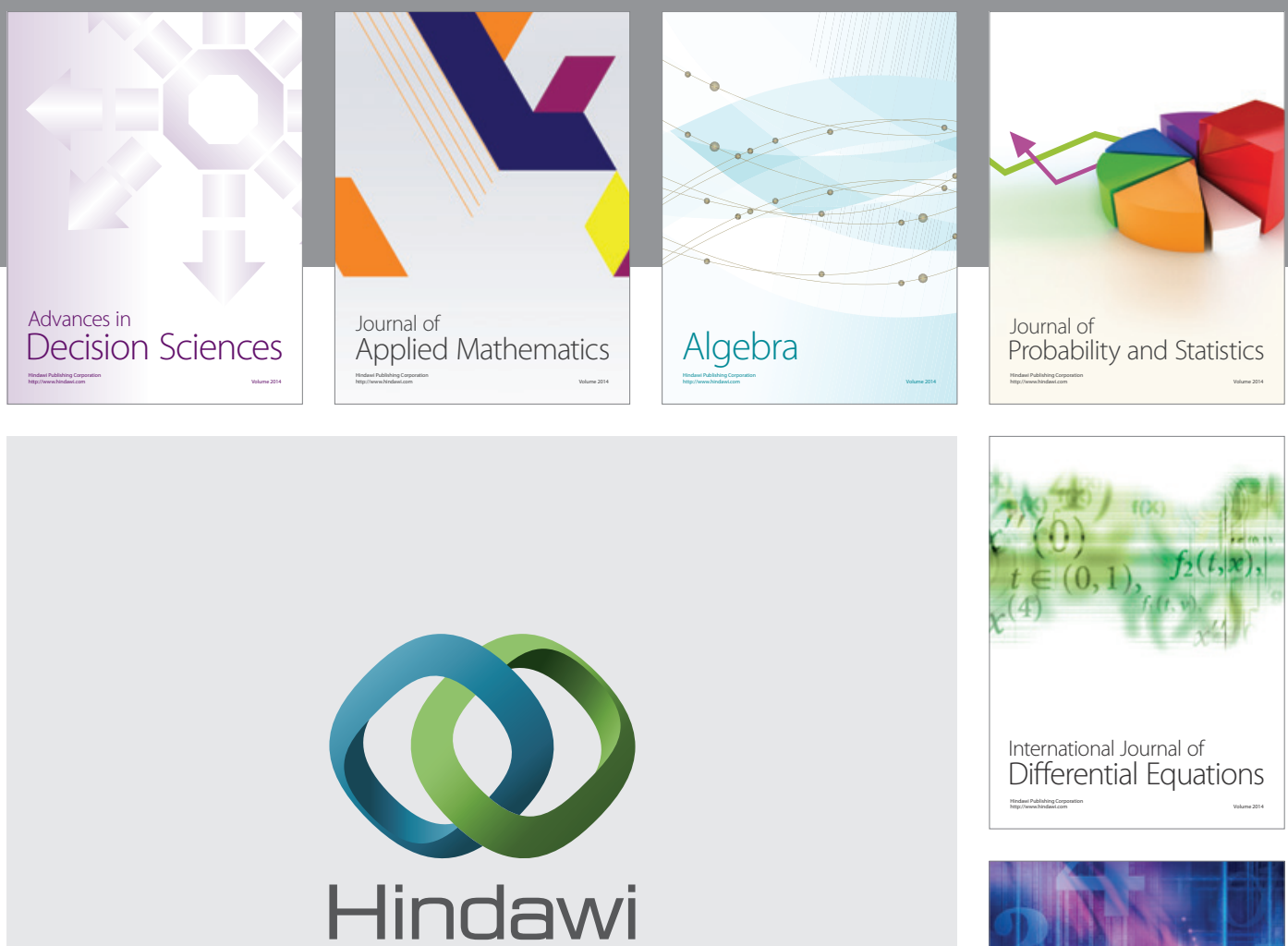

Submit your manuscripts at http://www.hindawi.com
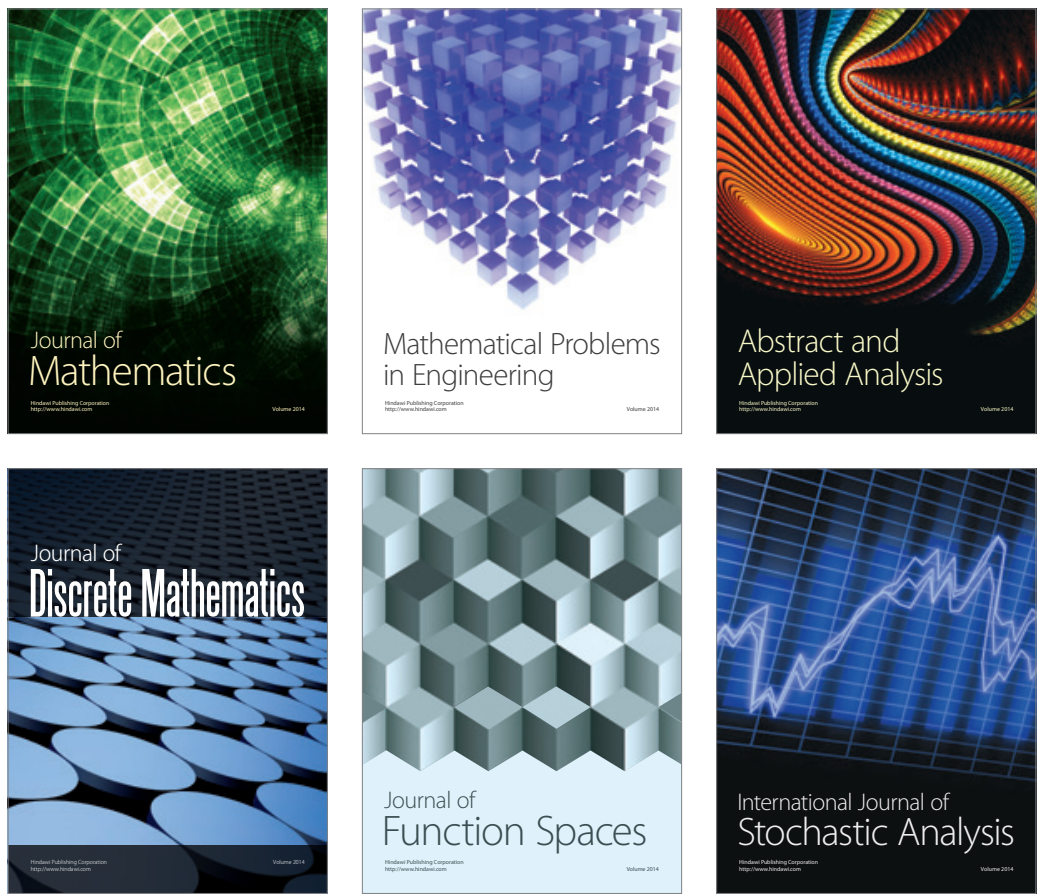

Journal of

Function Spaces

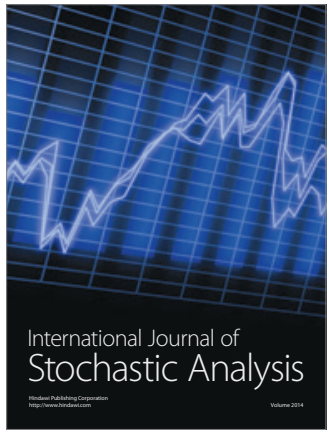

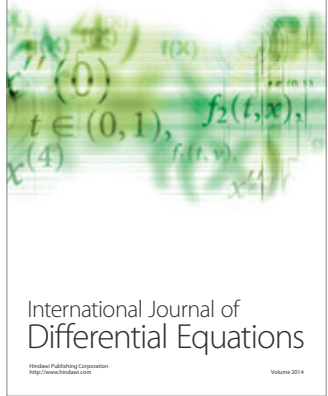
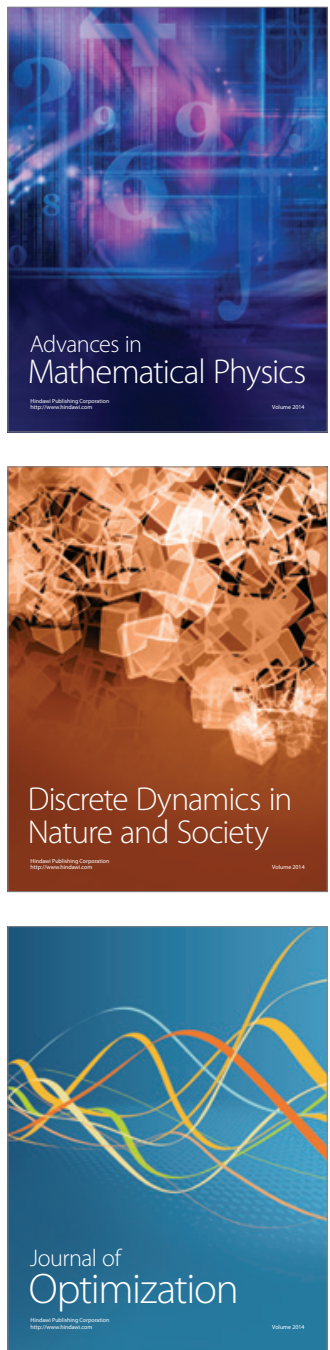